\title{
A Modified Galerkin Procedure with Hermite Cubics for Hyperbolic Problems
}

\author{
By Lars Wahlbin*
}

\begin{abstract}
The Galerkin method, modified to include a term of artificial viscosity type, is applied to model problems for linear and quasilinear hyperbolic systems. Asymptotic error estimates are derived.
\end{abstract}

1. Introduction. When solving hyperbolic problems numerically by finite-difference methods, one sometimes introduces, for various reasons, artificial viscosity. In this note we consider examples of the artificial viscosity technique applied to the numerical solution of hyperbolic problems by the Galerkin method.

Consider the 1-periodic initial-value problem for the real vector function $u=$ $u(x, t)$ with components $u^{(i)}(x, t), i=1, \ldots, N$, given by

$$
\begin{cases}u_{t}+A(x) u_{x}=0, & x \in R, 0<t \leqslant T, \\ u(x, 0)=u_{0}(x), & x \in R .\end{cases}
$$

Here the real $N \times N$ matrix $A(x)$ is 1-periodic and symmetric, and furthermore satisfies

$$
A^{2}(x) \geqslant C_{1}>0, \quad x \in R .
$$

Let $S_{h}$ be a space of periodic piecewise polynomial functions on a uniform mesh of size $h(h=1 / n, n=1,2, \ldots)$, and let $S_{h}=\left(S_{h}\right)^{N}$. Define a continuous-in-time Galerkin approximation to the solution of $(1.1)$ as a differentiable map $U:[0, T] \rightarrow S_{h}$ such that

$$
\left\{\begin{array}{l}
\left(U_{t}+A(x) U_{x}, \chi\right)+\widetilde{C} h^{\sigma}\left(d^{j} U / d x^{j}, d^{j} \chi / d x^{j}\right)=0, \quad \chi \in S_{h}, \\
U(0) \in S_{h} .
\end{array}\right.
$$

Here $(v, w)=\int_{0}^{1}\left(\sum_{i=1}^{N} v^{(i)}(x) w^{(i)}(x)\right) d x, \widetilde{C}$ denotes a positive constant which is independent of $h$, and it is assumed that $S_{h} \subset C^{j-1}(R)$.

In this note we shall for brevity treat only a special case of (1.3); in order to handle other cases, the appropriate modifications in the proof are reasonably straightforward. To motivate this special case, consider for a moment the ordinary Galerkin method, i.e., (1.3) with $\widetilde{C}=0$, from the point of view of asymptotic error estimates in $L^{2}$. For $B$-splines the ordinary Galerkin method gives optimal (best possible for the space in which the approximation is sought) asymptotic rate of convergence. In contrast, using the space of Hermite cubics, Dupont [2] has shown that the ordinary Galerkin method gives asymptotic rate of convergence $h^{3}$ and not better, in general,

Received November 13, 1974.

AMS (MOS) subject classifications (1970). Primary 65 N 30.

*This work was supported in part by the National Science Foundation. 
whereas the best possible rate is, a priori, $h^{4}$. We ask then whether for Hermite cubics, the method (1.3) with $\widetilde{C}>0$ and $j=1$ or 2 can give higher asymptotic rate of convergence for suitable $\sigma$. The method of proof below gives the best asymptotic rate, $h^{3.5}$, for $j=2$ and $\sigma=3.5$, and some simple numerical experiments by the author indicate that this is the best one can obtain asymptotically, although for smooth solutions and "reasonable" $h$ the ordinary Galerkin method may give smaller error. Hence, only the case mentioned above for the equation (1.1) will be treated. In Section 4 a similar example in a simple quasilinear situation will be considered. For related material see Dendy [1] and Wahlbin [4] where computationally more complicated Galerkin methods with optimal asymptotic rate of convergence for any space $S_{h}$ are discussed.

In the rest of this note, $S_{h}$ shall denote the space of periodic Hermite piecewise cubic functions, i.e.,

$$
\begin{aligned}
S_{h}= & \left\{\chi(x), x \in[0,1): \text { the periodic extension of } \chi \text { lies in } C^{1}(R)\right. \\
& \text { and } \left.\left.\chi\right|_{(i h,(i+1) h)} \text { is a polynomial of degree } \leqslant 3 \text { for } i=0, \ldots, n-1\right\} .
\end{aligned}
$$

We shall prove the following asymptotic error estimate.

THEOREM 1.1. Consider the problem (1.1) and its semidiscrete analogue (1.3) with $j=2$ and $\sigma=3.5$, where $A(x)$ is periodic, symmetric, belongs to $C^{2}(R)$ and satisfies (1.2). Assume that initial data for the Galerkin process are chosen such that with $C_{2}$ a constant,

$$
\left\|u_{0}-U(0)\right\|_{L^{2}} \leqslant C_{2} h^{3.5}
$$

Then there exists a constant $C_{3}=C_{3}\left(\widetilde{C}, C_{1}, C_{2},\|A\|_{C^{2}}\right)$ such that

$$
\|u-U\|_{L^{\infty}\left(0, T: L^{2}\right)} \leqslant h^{3.5} C_{3}\left\{1+\|u\|_{L^{\infty}\left(0, T: H_{4}\right)}+\left\|u_{t}\right\|_{L^{2}\left(0, T: H_{4}\right)}\right\} .
$$

As an example of generalizations of this result to other spaces of piecewise polynomials, consider such of degree $2 \mu-1$ which are in $C^{\mu-1}(\mu=2$ gives the Hermite cubics). With $j=\mu$ and $\sigma=2 \mu-1 / 2$, one then obtains, under appropriate assumptions, an asymptotic rate of convergence $h^{2 \mu-1 / 2}$.

After introducing notation and some preliminaries in Section 2, Theorem 1.1 will be proved in Section 3. In Section 4 the quasilinear example will be treated. This includes the problem (1.1) when (1.2) is not fulfilled.

2. Preliminaries. Throughout this note, $C$ will denote a positive constant not necessarily the same at each occurrence unless subindexed.

We first define some function spaces. For $D \subset R^{d}$ and $r$ a nonnegative integer, let $C^{r}(\bar{D})$ be the space of functions with $r$ continuous derivatives on $\bar{D}$, and with norm $\|\cdot\|_{C^{r}(\bar{D})}$ taken as the maximum over $\bar{D}$ of derivatives of orders up to $r$. For vectorvalued functions let the norm be the maximum of the norms of the components. The symbol $C^{r}$ will denote the space $C^{r}\left(R^{1}\right)$ with each component 1-periodic.

Furthermore, $H_{r}$ will denote the space of $N$-vector functions $v(x)$ where each component $v^{(i)}(x)$ is 1 -periodic and has $r$ derivatives in $L^{2}\left(R^{1}\right)$ locally, and with norm $\|v\|_{r}$ $=\left(\sum_{j=0}^{r}\left(d^{j} v / d x^{j}, d^{j} v / d x^{j}\right)\right)^{1 / 2}$ where 


$$
(v, w)=\int_{0}^{1}\left(\sum_{i=1}^{N} v^{(i)}(x) w^{(i)}(x)\right) d x .
$$

In particular, $L^{2}=H_{0}$ with norm $\|\cdot\|=\|\cdot\|_{0}$.

For negative integers $r$ define the norm $\|\cdot\|_{r}$ by duality over the $L^{2}$ inner product (2.1) so that for $v \in L^{2}$ say,

$$
\|v\|_{r}=\sup \left\{|(v, w)| /\|w\|_{-r}: w \in H_{-r},\|w\|_{-r} \neq 0\right\} .
$$

For a function $v(x, t)$ with $v(\cdot, t)$ in $H_{r}$ for each $t$, let

$$
\|v\|_{L^{p}\left(0, T: H_{r}\right)}=\|\| v(\cdot, t)\left\|_{r}\right\|_{L^{p}(0, T)} \text { for } 1 \leqslant p \leqslant \infty .
$$

We next collect two well-known approximation results for the Hermite spaces $S_{h}$.

Lemma 2.1. Let $0 \leqslant r \leqslant 4$. There exists a constant $C$, independent of $h$, such that for $v \in H_{r}$ there exists $\chi \in S_{h}$ such that for $-2 \leqslant l \leqslant \min (2, r)$,

$$
\|v-\chi\|_{l} \leqslant C h^{r-l}\|v\|_{r} .
$$

Lemma 2.2. Let $G(x)$ be a periodic matrix function in $C^{3}$. There exists a constant $C$, independent of $h$, such that for $\chi \in S_{h}$ there exists $\psi \in S_{h}$ such that for $0 \leqslant r \leqslant 2$,

$$
\|G(x) \chi-\psi\|_{r} \leqslant C h\|\chi\|_{r}
$$

Finally, we note the following elementary result.

LEMmA 2.3 (INVERSE PROPERTY). There exists a constant $C$, independent of $h$, such that for $\chi \in S_{h}$,

$$
\left\|d^{r} \chi / d x^{r}\right\| \leqslant C h^{-r+s}\left\|d^{s} \chi / d x^{s}\right\| \quad \text { for } 0 \leqslant s \leqslant r \leqslant 2,
$$

and

$$
\left\|d^{r} \chi / d x^{r}\right\|_{C^{0}} \leqslant h^{-1 / 2}\left\|d^{r} \chi / d x^{r}\right\| \quad \text { for } r=0,1 .
$$

3. Proof of Theorem 1.1. We shall compare the Galerkin solution $U(t)$ to a projection $W(t)$ of the solution of (1.1) into $S_{h}$, defined with $\rho=u-W$ by

$$
K(\rho, \chi)+\left(A(x) \rho_{x}, \chi\right)+\widetilde{C}^{3.5}\left(\rho_{x x}, \chi_{x x}\right)=0, \quad \chi \in S_{h} .
$$

Here $K$ is a sufficiently large constant which can be estimated from the proof of Lemma 3.1 below. For the error in this projection we have

Lемма 3.1. Let the hypotheses of Theorem 1.1 hold, and let $K$ be a sufficiently large constant. Then there exists a constant $C=C\left(\widetilde{C}, C_{1},\|A\|_{C^{2}}\right)$ such that for each fixed $t \in[0, T]$,

$$
\|\rho\| \leqslant C h^{3.5}\|u\|_{4}, \quad\left\|\rho_{t}\right\| \leqslant C h^{3.5}\left\|u_{t}\right\|_{4} .
$$

Proof. Let $\widetilde{C}=C_{1}=1$ for simplicity. It suffices to prove the first inequality of (3.2) since the second will follow by the same method of proof upon differentiating (3.1) with respect to time.

By the symmetry of $A(x)$ we have for $K$ large and $\chi \in S_{h}$, 


$$
\begin{aligned}
\|\rho\|^{2}+ & h^{3.5}\left\|\rho_{x x}\right\|^{2} \\
\leqslant & K(\rho, u-\chi)+\left(A(x) \rho_{x}, u-\chi\right)+h^{3.5}\left(\rho_{x x},(u-\chi)_{x x}\right) \\
\leqslant & K\|\rho\|\|u-\chi\|+C\|\rho\|_{2}\|u-\chi\|_{-1}+h^{3.5}\left\|\rho_{x x}\right\|\|u-\chi\|_{2} \\
\leqslant & 1 / 2\|\rho\|^{2}+1 / 2 h^{3.5}\left\|\rho_{x x}\right\|^{2} \\
& +C\left\{\|u-\chi\|^{2}+h^{-3.5}\|u-\chi\|_{-1}^{2}+h^{3.5}\|u-\chi\|_{2}^{2}\right\} .
\end{aligned}
$$

Choosing $\chi$ by Lemma 2.1, we obtain

$$
\|\rho\| \leqslant C h^{3.25}\|u\|_{4}, \quad\|\rho\|_{2} \leqslant C h^{1.5}\|u\|_{4} .
$$

Next use duality. Let $f$ be the solution of the 1-periodic problem $K f-(A f)_{x}+$ $h^{3.5} f_{x x x x}=\rho$. Using (1.2), it follows that

$$
\|f\|_{1} \leqslant C\|\rho\|, \quad h^{3.5}\|f\|_{4} \leqslant C\|\rho\| .
$$

For $\chi \in S_{h}$ we have

$$
\begin{aligned}
\|\rho\|^{2} & =K(\rho, f-\chi)+\left(A \rho_{x}, f-\chi\right)+h^{3.5}\left(\rho_{x x},(f-\chi)_{x x}\right) \\
& \leqslant K\|\rho\|\|f-\chi\|+C\|\rho\|_{2}\|f-\chi\|_{-1}+h^{3.5}\|\rho\|_{2}\|f-\chi\|_{2},
\end{aligned}
$$

and by Lemma 2.1 and (3.3) this gives

$$
\|\rho\|^{2} \leqslant C\left\{h^{3.25+1}\|f\|_{1}+h^{1.5+2}\|f\|_{1}+h^{3.5+1.5+2}\|f\|_{4}\right\}\|u\|_{4} .
$$

Then (3.4) implies that $\|\rho\|^{2} \leqslant C h^{3.5}\|\rho\|\|u\|_{4}$ which proves the lemma.

We now prove Theorem 1.1. Let $\widetilde{C}=C_{1}=C_{2}=1$ for simplicity and put $\theta=$ $U-W$. Then

$$
\left(\theta_{t}+A(x) \theta_{x}, \chi\right)+h^{3.5}\left(\theta_{x x}, \chi_{x x}\right)=\left(\rho_{t}, \chi\right)+K(\rho, \chi)-h^{3.5}\left(u_{x x x x}, \chi\right) .
$$

Let $\chi=\theta$. By Lemma 3.1 we obtain

$$
\frac{1}{2} \frac{d}{d t}\|\theta\|^{2} \leqslant C\left\{\|\theta\|^{2}+h^{7}\left(\|u\|_{4}^{2}+\left\|u_{t}\right\|_{4}^{2}\right)\right\} .
$$

Since by (1.4) and Lemma 3.1

$$
\|\theta(0)\| \leqslant\left\|u_{0}-U(0)\right\|+\|\rho(0)\| \leqslant C h^{3.5}\left(1+\left\|u_{0}\right\|_{4}\right),
$$

Gronwall's lemma implies that

$$
\|\theta\|_{L^{\infty}\left(0, T: L^{2}\right)} \leqslant C h^{3.5}\left\{1+\left\|u_{0}\right\|_{4}+\|u\|_{L^{2}\left(0, T: H_{4}\right)}+\left\|u_{t}\right\|_{L^{2}\left(0, T: H_{4}\right)}\right\} .
$$

By Lemma 3.1 and the triangle inequality, this proves Theorem 1.1.

4. Symmetrizable Quasilinear Systems. Consider the 1-periodic problem for the $N$-vector function $u(x, t)$ given by

$$
\begin{cases}u_{t}+A(u) u_{x}=0, & x \in R, 0 \leqslant t \leqslant T, \\ u(x, 0)=u_{0}(x), & x \in R .\end{cases}
$$

Assume that this problem has a unique solution such that 


$$
u \in L^{\infty}\left(0, T: H_{4}\right), \quad u_{t} \in L^{2}\left(0, T: H_{4}\right) .
$$

Note that this implies by Sobolev's theorem that $u(\cdot, t) \in \mathrm{C}^{3}$.

Let $\left[y_{0}^{(i)}, y_{1}^{(i)}\right]=\left\{u^{(i)}(x, t), x \in R, 0 \leqslant t \leqslant T\right\}$ and let for $\delta>0, M_{\delta}=$ $\Pi_{i=1}^{N}\left[y_{0}^{(i)}-\delta, y_{1}^{(i)}+\delta\right] \subset R^{N}$. The matrix function $A(\cdot)$ is assumed to satisfy the following:

(i) There exists $\delta>0$ such that $A(\cdot) \in C^{3}\left(M_{\delta}\right)$.

(ii) There exists a symmetric matrix function $D(\cdot) \in C^{3}\left(M_{\delta}\right)$ with

$$
\begin{aligned}
& D(q) \geqslant C_{4}>0, \quad q \in M_{\delta}, \\
& D(q) A(q)=S(q), \quad q \in M_{\delta},
\end{aligned}
$$

where $S(q)$ is symmetric.

Pose the Galerkin problem of finding $U:[0, T] \rightarrow S_{h}$ such that

$$
\left\{\begin{array}{l}
\left(U_{t}+A(U) U_{x}, \chi\right)+\widetilde{C} h^{10 / 3}\left(U_{x x}, \chi_{x x}\right)=0, \quad \chi \in S_{h}, \\
U(0) \in S_{h} .
\end{array}\right.
$$

We have the following asymptotic error estimate.

THEOREM 4.1. Consider the problem (4.1) and its semidiscrete analogue (4.5). Assume that (4.2) and (i), (ii) are satisfied, and that there exists a constant $C_{5}$ such that

$$
\left\|u_{0}-U(0)\right\|_{2} \leqslant C_{5} h^{10 / 3}
$$

Then there exist positive constants $h_{0}$ and $C_{6}$, depending on $\widetilde{C}, \delta,\|A\|_{C^{3}\left(M_{\delta}\right)}, C_{4}, C_{5}$, $\|u\|_{L^{\infty}\left(0, T: H_{4}\right)}$, and $\left\|u_{t}\right\|_{L^{2}\left(0, T: H_{4}\right)}$ such that for $h \leqslant h_{0}$, the Galerkin solution exists for $0 \leqslant t \leqslant T$ and

$$
\|u-U\|_{L^{\infty}\left(0, T: L^{2}\right)} \leqslant C_{6} h^{10 / 3} .
$$

The method (4.5) generalizes to spaces of piecewise polynomials of degree $2 \mu-1$ which are in $C^{\mu-1}$ with the viscosity term taken as $\widetilde{C} h^{2 \mu-2 / 3}\left(d^{\mu} U / d x^{\mu}, d^{\mu} \chi / d x^{\mu}\right)$ to give an asymptotic rate of convergence $h^{2 \mu-2 / 3}$. For an example of the ordinary Galerkin method in a similar situation, see Dupont [3] .

Proof of Theorem 4.1. We shall compare the Galerkin solution to the projection $W(t)$ of the solution of (4.1) into $S_{h}$ given, with $\rho=u-W$, by the requirement $(\rho, \chi)=0$ for $\chi \in S_{h}$. As is well known,the following estimates hold:

$$
\begin{aligned}
& \|\rho\|_{r} \leqslant C h^{4-r}\|u\|_{4}, \quad r=-1,0,1,2, \\
& \left\|\rho_{t}\right\| \leqslant C h^{4}\left\|u_{t}\right\|_{4} .
\end{aligned}
$$

Let $\widetilde{C}=C_{4}=C_{5}=1$ for simplicity, and set $\theta=U-W$.

We shall assume a priori that $U$ exists for $0 \leqslant t \leqslant t_{0}$, that

$$
U(t)(x) \in M_{\delta}, \quad 0 \leqslant t \leqslant t_{0},
$$

and that

$$
\left\|\theta_{x}\right\|_{c^{0}} \leqslant 1, \quad 0 \leqslant t \leqslant t_{0}
$$


Note that $\|\rho\|_{C 0}+\left\|\rho_{x}\right\|_{C 0} \leqslant C\|\rho\|_{2} \leqslant C h^{2}$, and hence $W \in M_{\delta / 4}$ for $h$ small. The assumption (4.11) implies then that

$$
\left\|U_{x}\right\|_{C^{0}} \leqslant C, \quad 0 \leqslant t \leqslant t_{0} .
$$

Also note that $U(0)(x) \in M_{\delta / 2}$ for $h$ sriall, and hence we may assume that $t_{0} \geqslant t_{0}(h)>0$ by well-known local existence theorems for ordinary differential equations.

We have for $\chi \in S_{h}$ that

$$
\begin{aligned}
\left(\theta_{t}+A(u) \theta_{x}, \chi\right)+h^{10 / 3}\left(\theta_{x x}, \chi_{x x}\right) \\
=\left((A(u)-A(W)+A(W)-A(U)) U_{x}, \chi\right)+\left(\rho_{t}, \chi\right) \\
\quad+\left(A(u) \rho_{x}, \chi\right)+h^{10 / 3}\left(\rho_{x x}, \chi_{x x}\right)-h^{10 / 3}\left(u_{x x x x}, \chi\right) .
\end{aligned}
$$

Let first $\chi=D(u) \theta+R$, where by Lemma 2.2 we may assume that

$$
\|R\| \leqslant C h\|\theta\|, \quad\|R\|_{2} \leqslant C h\|\theta\|_{2} .
$$

To handle various terms in (4.13) we note in particular (4.15)-(4.19) below. By (4.4) we have

$$
\begin{aligned}
\left(\theta_{t}+\right. & \left.A(u) \theta_{x}, D(u) \theta\right) \\
& =\frac{1}{2} \frac{d}{d t}\left\|D^{1 / 2} \theta\right\|^{2}-\left(D(u)_{t} \theta, \theta\right)+\left(S(u) \theta_{x}, \theta\right) \\
& =\frac{1}{2} \frac{d}{d t}\left\|D^{1 / 2} \theta\right\|^{2}-\left(D(u)_{t} \theta, \theta\right)-\frac{1}{2}\left(S(u)_{x} \theta, \theta\right) .
\end{aligned}
$$

Further, by (2.2),

$$
h^{10 / 3}\left(\theta_{x x}, D \theta_{x x}\right) \leqslant h^{10 / 3}\left(\theta_{x x},(D \theta)_{x x}\right)+C\left\|D^{1 / 2} \theta\right\|^{2} .
$$

From the a priori assumption (4.10) and from (4.12) we see that

$$
\left|\left((A(u)-A(W)+A(W)-A(U)) U_{x}, \chi\right)\right| \leqslant C\left(\|\rho\|+\left\|D^{1 / 2} \theta\right\|\right)\|\chi\| .
$$

By (4.8) we have

$$
\left|\left(A(u) \rho_{x}, \chi\right)\right| \leqslant C\|\rho\|_{-1}\|\chi\|_{2} \leqslant C h^{5}\|\chi\|_{2} .
$$

Also,

$$
\left|h^{10 / 3}\left(\rho_{x x}, \chi_{x x}\right)\right| \leqslant C h^{5}\|x\|_{2}
$$

The results (4.13)-(4.19) imply, after some further simplification and using also (4.9),

$$
\frac{1}{2} \frac{d}{d t}\left\|D^{1 / 2} \theta\right\|^{2}+h^{10 / 3}\left\|D^{1 / 2} \theta_{x x}\right\|^{2} \leqslant C\left(\left\|D^{1 / 2} \theta\right\|^{2}+h^{20 / 3}\right)+\frac{1}{2} h^{2}\left\|\theta_{t}\right\|^{2} .
$$

Next let $\chi=\theta_{t}$ in (4.13). We have then

$$
\frac{1}{2}\left\|\theta_{t}\right\|^{2}+\frac{1}{2} h^{10 / 3} \frac{d}{d t}\left\|\theta_{x x}\right\|^{2} \leqslant C\left(\left\|\theta_{x}\right\|^{2}+\|\theta\|^{2}+h^{6}\right) .
$$

Inserting this into (4.20) and using (2.2), it follows that

$$
\frac{1}{2} \frac{d}{d t}\left(\left\|D^{1 / 2} \theta\right\|^{2}+h^{16 / 3}\left\|\theta_{x x}\right\|^{2}\right) \leqslant C\left(\left\|D^{1 / 2} \theta\right\|^{2}+h^{20 / 3}\right) .
$$


By (4.6), (4.8) and (2.2) we see that $\left\|D^{1 / 2} \theta(0)\right\|^{2}+h^{16 / 3}\left\|\theta_{x x}(0)\right\|^{2} \leqslant C h^{20 / 3}$, and (4.21) then gives via Gronwall's lemma and using (4.3),

$$
\|\theta\| \leqslant C_{7} h^{10 / 3}, \quad 0 \leqslant t \leqslant t_{0} .
$$

By (2.2) and (2.3) this implies $\|\theta\|_{C^{0}} \leqslant C h^{17 / 6}$. Since $W \in M_{\delta / 4}$ we have for $h \leqslant h_{0}$ with $h_{0}$ sufficiently small the following sharpenings of the a priori assumptions (4.10) and (4.11), viz., that for $0 \leqslant t \leqslant t_{0}, U(t)(x) \in M_{\delta / 2}$ and $\left\|\theta_{x}\right\|_{c^{0}} \leqslant C_{8} h^{11 / 6}$. Tracing the dependence of the constants above, it is seen that $h_{0}$ does not depend on $t_{0}$ for $t_{0} \leqslant T$; and the constants $C_{7}$ and $C_{8}$ do not depend on $h$ nor $t_{0}$ for $h \leqslant h_{0}$ and $t_{0} \leqslant T$. Taking $h_{0}$ such that $C_{8} h_{0}^{11 / 6} \leqslant 1 / 2$, it follows from local existence theorems for ordinary differential equations that existence of $U(t)$ and (4.10), (4.11) can be asserted for $0 \leqslant t \leqslant t_{0}+\epsilon(h)$, where $\epsilon(h)>0$ does not depend on $t_{0}$ for $t_{0} \leqslant T$. Hence, by iteration, the Galerkin solution exists on $[0, T]$ and satisfies (4.22).

The desired result (4.7) now follows from (4.22) and (4.8) via the triangle inequality.

Department of Mathematics

University of Chicago

Chicago, Illinois 60637

Department of Mathematics

Cornell University

Ithaca, New York 14850

1. J. E. DENDY, "Two methods of Galerkin type achieving optimum $L^{2}$-accuracy for first order hyperbolics," SIAM J. Numer. Anal., v. 11, 1974, pp. 637-653.

2. T. DUPONT, "Galerkin methods for first order hyperbolics: an example," SIAM J. Numer. Anal., v. 10, 1973, pp. 890-899.

3. T. DUPONT, "Galerkin methods for modeling gas pipelines," Constructive and Computational Methods for Differential and Integral Equations, D. L. Colton and R. P. Gilbert (Editors), Lecture Notes in Math., vol 430, Springer-Verlag, New York, 1974, pp. 112-130.

4. L. WAHLBIN, "A dissipative Galerkin method applied to some quasi-linear hyperbolic equations," Rev. Française Automat. Informat. Recherche Opérationelle Sér. Verte, v. 8, 1974, pp. 109-117. 